Case Report

\title{
Treatment of Recurrent Posttransplant Lymphoproliferative Disorder with Autologous Blood Stem Cell Transplant
}

\author{
Bharat Malhotra, ${ }^{1}$ Ahmad K. Rahal, ${ }^{1}$ Hussam Farhoud, ${ }^{1,2}$ \\ Dennis F. Moore Jr., ${ }^{1,3}$ and K. James Kallail ${ }^{1}$ \\ ${ }^{1}$ Department of Internal Medicine, University of Kansas School of Medicine-Wichita, 1010 N. Kansas Street, Wichita, KS 67214, USA \\ ${ }^{2}$ Heartland Cardiology, Heartland West, 9000 W. Central, Wichita, KS 67212, USA \\ ${ }^{3}$ Cancer Center of Kansas, 818 N. Emporia, No. 403, Wichita, KS 67214, USA
}

Correspondence should be addressed to Bharat Malhotra; drbharat25@gmail.com

Received 4 August 2015; Revised 13 October 2015; Accepted 5 November 2015

Academic Editor: Mohammed Rafique Moosa

Copyright (c) 2015 Bharat Malhotra et al. This is an open access article distributed under the Creative Commons Attribution License, which permits unrestricted use, distribution, and reproduction in any medium, provided the original work is properly cited.

\begin{abstract}
Background. Posttransplant lymphoproliferative disorders (PTLDs) occur after solid organ transplantation. Treatment guidelines include reduction in immunosuppression (RIS), radiation, rituximab, chemotherapy, and immunological agents. We present a rare case of recurrent diffuse large B-cell lymphoma presenting as a PTLD in a heart transplant patient treated with autologous blood stem cell transplant (ASCT) after failure of conventional therapy. Case Presentation. A 66-year-old male presented with a neck mass. He has a history of Hodgkin's disease status after staging laparotomy with splenectomy and heart transplantation due to dilated nonischemic cardiomyopathy 8 years prior to the development of PTLD. His examination was remarkable for right submandibular swelling. An excisional biopsy confirmed the diagnosis of diffuse large B-cell NHL. Patient received RIS, rituximab, chemotherapy, and radiation therapy with a complete remission. His lymphoma relapsed and he subsequently was treated with RICE salvage chemotherapy and consolidative high-dose chemotherapy with BEAC regimen followed by ASCT resulting in a complete remission. Conclusion. Patients with PTLD present a difficult therapeutic challenge. In this case, the patient's prior history of Hodgkin's disease, splenectomy, and a heart transplant appear to be unique features, the significance of which is unclear. ASCT might be a promising therapy for patients with relapsed or refractory PTLD.
\end{abstract}

\section{Introduction}

Posttransplant lymphoproliferative disorders (PTLDs) are a heterogeneous group of benign and malignant entities that occur in the setting of solid organ or allogeneic hematopoietic cell transplantation in the setting of immunosuppression [1]. PTLD is the most common cause of cancer-related mortality in patients with solid organ transplantation [2]. About 85\% of PTLDs in the United States are of B-cell origin. The optimal treatment of PTLD is not clearly defined due to a lack of randomized phase III trials. Current management options include reduction in immunosuppression (RIS), surgery, radiation, rituximab, chemotherapy, and antiviral and immunological agents. We present an unusual case of diffuse large B-cell type Non-Hodgkin's Lymphoma (NHL) presenting as a PTLD in an adult with a history of Hodgkin's disease, splenectomy, and heart transplant treated with autologous blood stem cell transplant (ASCT) after failure of conventional therapy.

\section{Case Presentation}

A 66-year-old male patient with a past medical history of dilated nonischemic cardiomyopathy status after heart transplant, immune thrombocytopenic purpura, hypertension, and dyslipidemia presented with a gradually enlarging right neck mass. On physical examination, there was a swelling in the right preauricular and submandibular lymph nodes extending into the cervical nodal area. Laboratory data showed mild leukocytosis but were otherwise unremarkable. His immunosuppressive medications included prednisone $10 \mathrm{mg}$ once daily, tacrolimus $1.5 \mathrm{mg}$ twice a day, and 


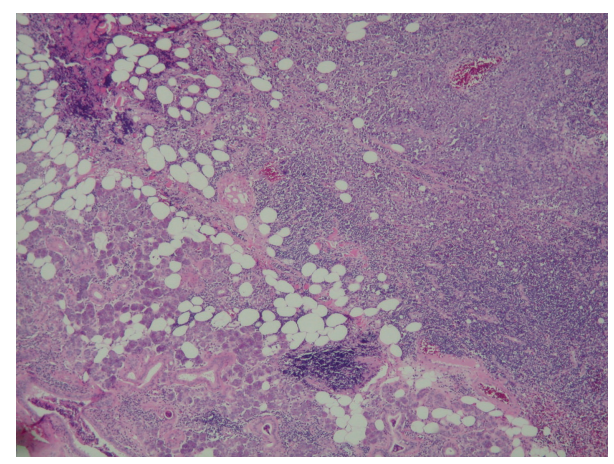

FIGURE 1: Hematoxylin and eosin stain showing salivary gland and lymphoid components. Small lymphocytes on the left and large lymphocytes on the right.

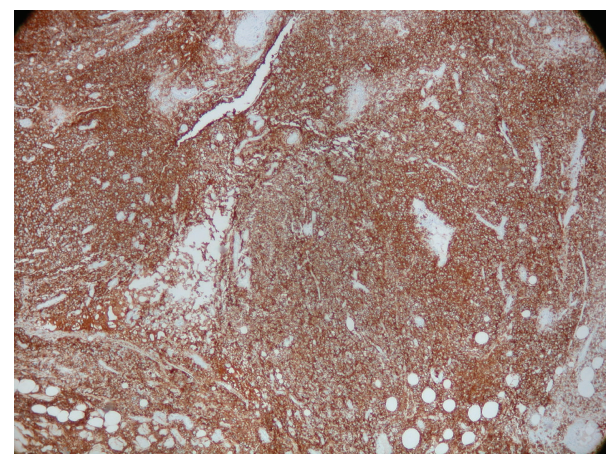

Figure 2: CD 20 immunostain showing 80-90\% large lymphoid cells.

mycophenolate $750 \mathrm{mg}$ twice a day. His workup led to a fine needle aspiration biopsy which was nondiagnostic. An excisional lymph node biopsy confirmed the diagnosis of diffuse large B-cell type NHL germinal center cell subtype (Figure 1). Immunohistochemical studies showed positive staining for CD20 (Figure 2) with high Ki-67 score of approximately $90 \%$ (Figure 3). Workup for his NHL included a computerized tomography (CT) scan of neck, chest, abdomen, and pelvis which showed lymphadenopathy in the right parotid and submandibular areas extending anteriorly toward the floor of the mouth, as well as in the right cervical region. Positron Emission Tomography (PET) scan showed corresponding hypermetabolic uptake in the neck. Bone marrow biopsy and aspiration were negative. Human immunodeficiency virus, Epstein-Barr virus (EBV), cytomegalovirus, and hepatitis panel were negative. The stage of his primary lymphoma was Ann Arbor Stage IA [3].

Per recommended guidelines for PTLD treatment [2], RIS was initiated. Prednisone was reduced from $10 \mathrm{mg}$ to $5 \mathrm{mg}$ daily, tacrolimus was reduced from $1.5 \mathrm{mg}$ to $0.5 \mathrm{mg}$ twice a day, and mycophenolate was stopped. There were no further changes in the immunosuppressive treatment during the investigational time, nor was there a change from tacrolimus to $\mathrm{m}$-Tor inhibitors. The patient received six cycles of chemotherapy with rituximab, cyclophosphamide, doxorubicin, vincristine, and prednisone $(\mathrm{R}+\mathrm{CHOP})$.

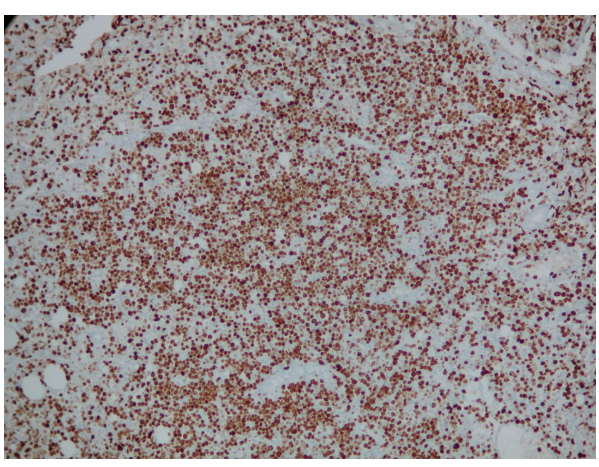

FIGURE 3: Ki-67 immunostain showing high proliferation rate.

Physical examination, complete blood count (CBC), complete metabolic panel (CMP), lactate dehydrogenase (LDH), CT scans, and PET-CT scans confirmed the patient to be in complete remission. Remission duration was brief, four weeks later with biopsy proving local relapse occurring. He received salvage chemotherapy with rituximab, ifosfamide, carboplatin, and etoposide (RICE) chemotherapy. Unfortunately, after 2 cycles of RICE the disease progressed and the mass had become ulcerated and necrotic. Repeat PET scan showed local relapse and he was treated with radiation therapy at standard dose plus fractionation. Despite an initial response, the mass progressed again during radiation therapy. Subsequent treatment with rituximab, gemcitabine, and oxaliplatin ( + GEMOX) resulted in a partial remission. With demonstrated chemosensitivity of his primary refractory disease, he proceeded with high-dose chemotherapy regimen of carmustine, etoposide, cytarabine, and cyclophosphamide (BEAC) with ASCT. Following the ASCT, the patient achieved a complete clinical remission and experienced no recurrence of the PTLD NHL.

\section{Discussion}

Posttransplantation lymphomas were first described in 1968 [4]. Patients with PTLD present a difficult clinical challenge. PTLD may develop at any time following organ transplantation; however, its risk is highest during the first year [1]. Incidence of PTLD after heart transplantation is around 1$6 \%$ [5]. The risk for PTLD can be reduced by limiting patient exposure to aggressive immunosuppressive regimens $[1,2]$. The pathogenesis of PTLD in most patients appears to be related to B-cell proliferation induced by infection with EBV in the setting of chronic immunosuppression and decreased T-cell immune surveillance [6]. It may be worth considering the additional impact on immune surveillance of a prior diagnosis of Hodgkin's lymphoma and splenectomy, as in this patient. The prognosis is favorable in early PTLD and poor in late PTLD.

The treatment of PTLD is a complex task requiring special considerations. The hematooncology subgroup of the British Committee for Standards in Hematology and the British Transplantation Society recently has made recommendations for the management of PTLD in adult recipients of solid 
organ transplants, based on literature data and the experience of PTLD specialists [2]. They did not include ASCT in their recommendations for treating PTLD. Treatment options of PTLD are RIS, rituximab, rituximab plus chemotherapy, surgery, radiotherapy, adoptive T-cell therapy, and antiviral and immunological agents [2]. Despite these treatments, the overall mortality of PTLD after solid organ transplantation is around $50 \%$ [7]. The rationale of using ASCT in our patient was due to failure of conventional chemotherapy and radiation therapy. The evidence for high-dose chemotherapy and stem cell transplant comes from the PARMA study [8]. Philip et al. [9] showed that, compared with conventional chemotherapy, treatment with high-dose chemotherapy and autologous bone marrow transplantation increases event-free and overall survival in patients with chemosensitive NonHodgkin's lymphoma in relapse.

With respect to the cardiac performance at remission and at follow-up, there was no evidence of allograft dysfunction determined by surveillance echocardiogram and cardiac catheterization with an ejection fraction of more than $55 \%$. Clinically, patient overall status after ASCT and on followup remained stable at New York Heart Association (NYHA) Functional Classification Classes I-II [10]. The patient did not have any signs of severe allograft rejection at any point and this was verified status after right ventricle endomyocardial biopsy. The worse rejection the patient had according to the International Society of Heart and Lung Transplantation (ISHLT) was Grade 1R [11-13]. Grade $1 \mathrm{R}$ is generally not treated unless there is concomitant hemodynamic dysfunction $[14,15]$. Our patient was always hemodynamically stable and was never treated for mild allograft rejection (Grade 1R). The follow-up time after ASCT was 17 months.

\section{Conclusion}

PTLD is a serious complication of solid organ transplantation, contributing significantly to morbidity and mortality in this patient group. To our knowledge, treatment with ASCT for PTLD following heart transplant has not been reported before. ASCT might be a promising therapy for PTLD after solid organ transplant that is resistant to recommended therapy, and data from future randomized clinical trials will be important.

\section{Conflict of Interests}

The authors declare that there is no conflict of interests regarding the publication of this paper.

\section{References}

[1] G. Végso, M. Hajdu, and A. Sebestyén, "Lymphoproliferative disorders after solid organ transplantation-classification, incidence, risk factors, early detection and treatment options," Pathology and Oncology Research, vol. 17, no. 3, pp. 443-454, 2011.

[2] A. Parker, K. Bowles, J. A. Bradley et al., "Management of posttransplant lymphoproliferative disorder in adult solid organ transplant recipient-BCSH and BTS Guidelines," British Journal of Haematology, vol. 149, no. 5, pp. 693-705, 2010.
[3] P. P. Carbone, H. S. Kaplan, K. Musshoff, D. W. Smithers, and M. Tubiana, "Report of the committee on Hodgkin's disease staging classification.," Cancer Research, vol. 31, no. 11, pp. 1860-1861, 1971.

[4] J. E. Murray, R. E. Wilson, N. L. Tilney et al., "Five years' experience in renal transplantation with immunosuppressive drugs: survival, function, complications, and the role of lymphocyte depletion by thoracic duct fistula," Annals of Surgery, vol. 168, no. 3, pp. 416-435, 1968.

[5] S. H. Swerdlow, S. A. Webber, A. Chadburn, and J. A. Ferry, "Post-transplant lymphoproliferative disorders," in WHO Classification of Tumors of Haematopoietic and Lymphoid Tissue, pp. 343-353, IARC, Lyon, France, 2008.

[6] D. W. Hanto, "Classification of Epstein-Barr virus-associated posttransplant lymphoproliferative diseases: implications for understanding their pathogenesis and developing rational treatment strategies," Annual Review of Medicine, vol. 46, pp. 381394, 1995.

[7] T. Haque, G. M. Wilkie, M. M. Jones et al., "Allogeneic cytotoxic T-cell therapy for EBV-positive posttransplantation lymphoproliferative disease: results of a phase 2 multicenter clinical trial," Blood, vol. 110, no. 4, pp. 1123-1131, 2007.

[8] T. Philip, F. Chauvin, J. Armitage et al., "Parma international protocol: pilot study of DHAP followed by involved-field radiotherapy and BEAC with autologous bone marrow transplantation," Blood, vol. 77, no. 7, pp. 1587-1592, 1991.

[9] T. Philip, C. Guglielmi, A. Hagenbeek et al., "Autologous bone marrow transplantation as compared with salvage chemotherapy in relapses of chemotherapy-sensitive non-Hodgkin's lymphoma," The New England Journal of Medicine, vol. 333, no. 23, pp. 1540-1545, 1995.

[10] The Criteria Committee of the New York Heart Association, Nomenclature and Criteria for Diagnosis of Diseases of the Heart and Great Vessels, Little, Brown and Company, Boston, Mass, USA, 9th edition, 1994.

[11] M. E. Billingham, N. R. Cary, M. E. Hammond et al., "A working formulation for the standardization of nomenclature in the diagnosis of heart and lung rejection: heart rejection study group. The International Society for Heart Transplantation," The Journal of Heart Transplantation, vol. 9, no. 6, pp. 587-593, 1990.

[12] G. L. Winters, C. C. Marboe, and M. E. Billingham, "The international society for heart and lung transplantation grading system for heart transplant biopsy specimens: clarification and commentary," Journal of Heart and Lung Transplantation, vol. 17, no. 8, pp. 754-760, 1998.

[13] S. Stewart, G. L. Winters, M. C. Fishbein et al., "Revision of the 1990 working formulation for the standardization of nomenclature in the diagnosis of heart rejection," Journal of Heart and Lung Transplantation, vol. 24, no. 11, pp. 1710-1720, 2005.

[14] G. L. Winters, E. Loh, and F. J. Schoen, "Natural history of focal moderate cardiac allograft rejection. Is treatment warranted?" Circulation, vol. 91, no. 7, pp. 1975-1980, 1995.

[15] J.-J. Lloveras, G. Escourrou, M. B. Delisle et al., "Evolution of untreated mild rejection in heart transplant recipients," Journal of Heart and Lung Transplantation, vol. 11, no. 4, part 1, pp. 751756, 1992. 


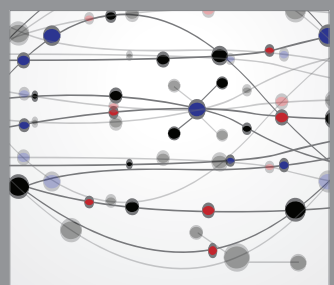

The Scientific World Journal
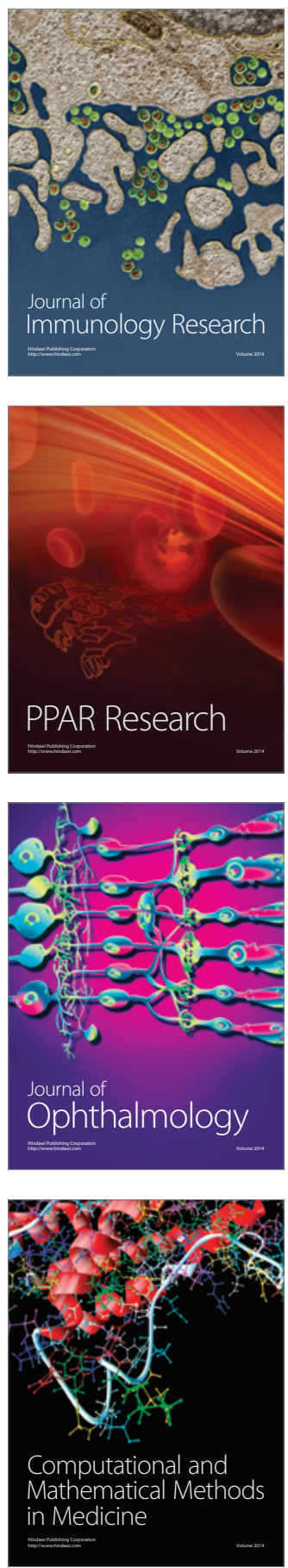

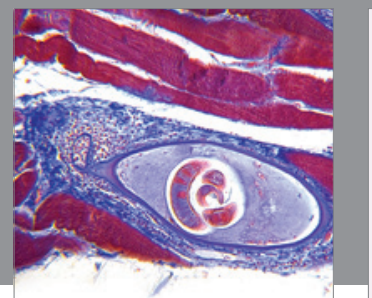

Gastroenterology

Research and Practice
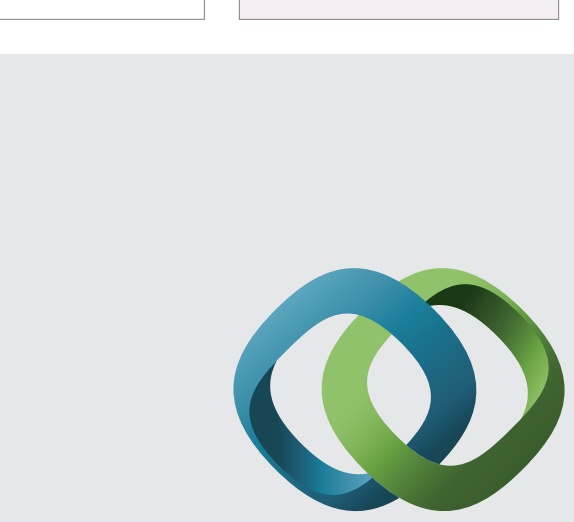

\section{Hindawi}

Submit your manuscripts at

http://www.hindawi.com
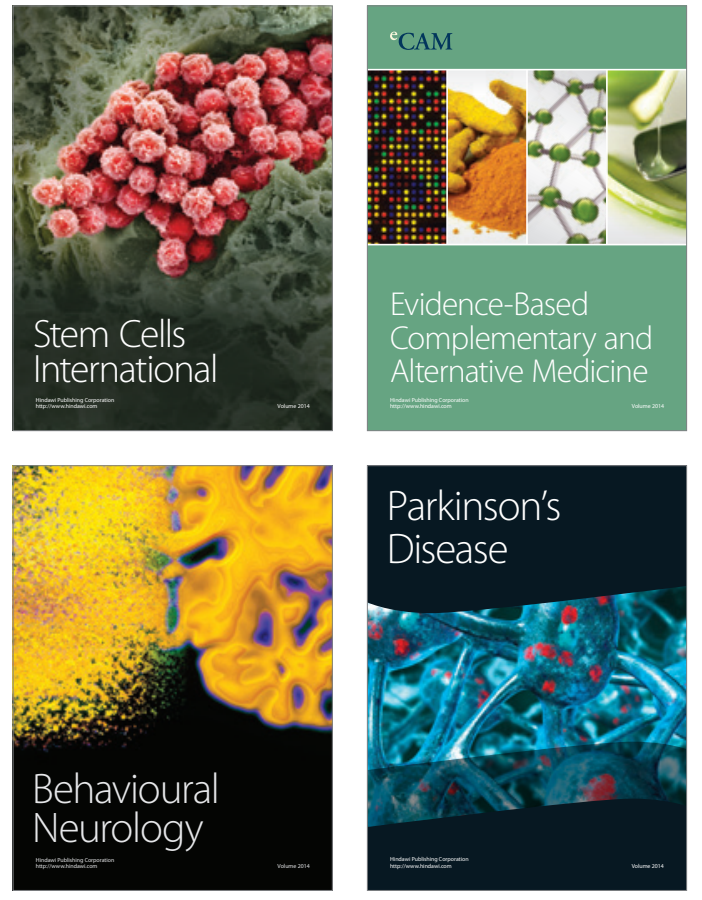
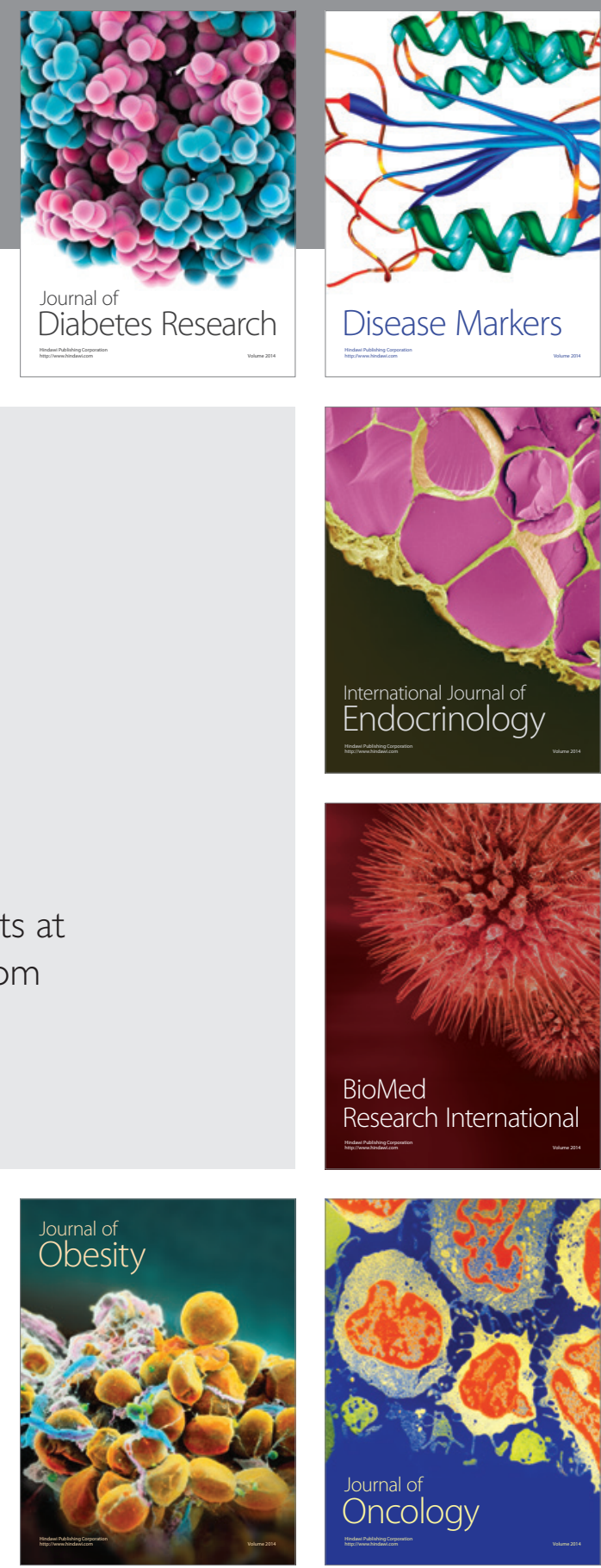

Disease Markers
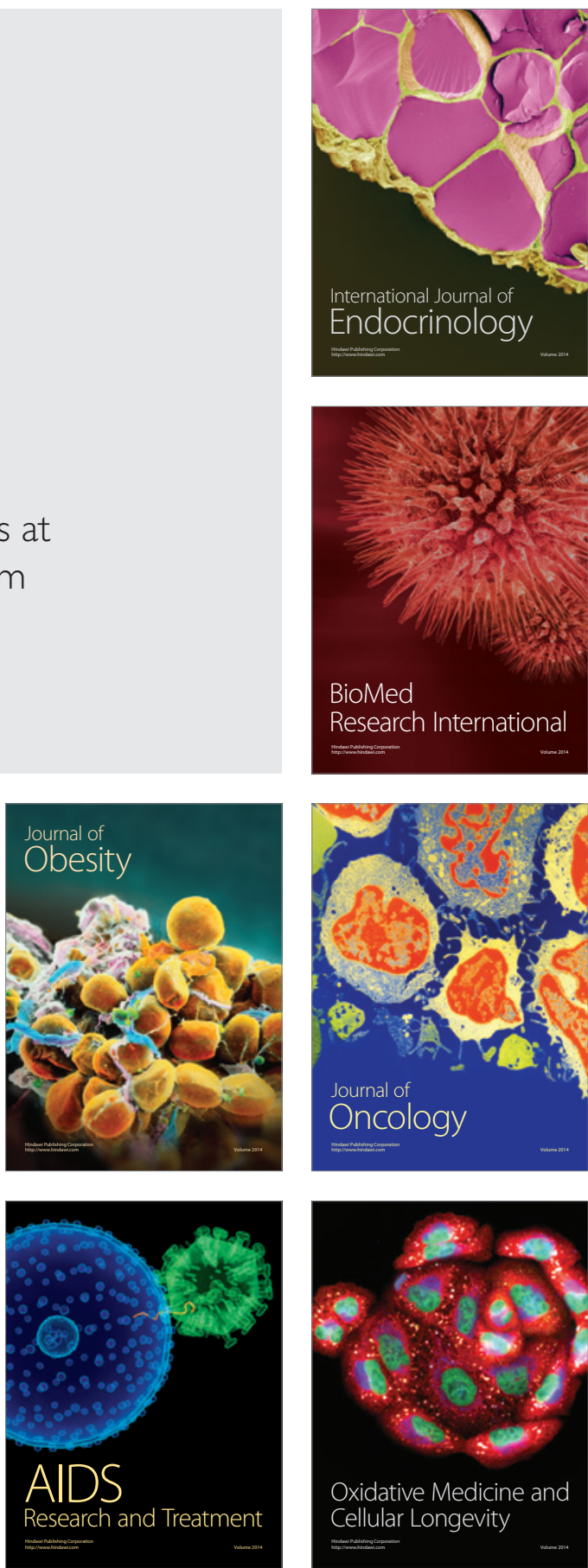